\section{Hunde schützen vor Sensibilisierung}

\author{
Hunde können nicht nur liebenswerte Spielgefährten für Kinder \\ sein, der enge Kontakt mit ihnen schützt möglicherweise auch vor \\ Sensibilisierungen - so zumindest das neueste Ergebnis zum alten \\ Thema Tierhaltung und Allergieentwicklung.
}

B isherige Studien zum Allergierisiko in Abhängigkeit von der Exposition gegenüber Hunden in der Kindheit hatten widersprüchliche Ergebnisse erbracht. Nun wurden die Daten zweier großer deutschen Kohortenstudien GINI und LISA - mit insgesamt 3.155 Kindern gemeinsam ausgewertet. In beiden Studien waren der Kontakt zu Hunden, allergische Symptome sowie vom Arzt diagnostizierte allergische Erkrankungen $\mathrm{zu}$ verschiedenen Zeitpunkten mit Fragebögen erfasst worden. Zusätzlich hatten die Untersucher spezifische IgE-Antikörper der Kinder im Alter von sechs Jahren bestimmt und Hausstaub zur Ermittlung der Endotoxinbelastung gesammelt, als die Kinder drei Monate alt waren. Im Mittel wurde in beiden Studien in jedem zehnten Haushalt ein Hund gehalten, nur im Hochrisikoarm der GINI-Studie, in dem 90\% der El- tern eine Allergie in der Anamnese aufwiesen, lag der Anteil mit 8,5\% etwas niedriger.

Mit einem Hund als Familienmitglied entwickelten die Kinder in beiden Kohorten seltener eine Sensibilisierung auf inhalative Allergene als Kinder, die ohne Hund aufwuchsen. Der Effekt war

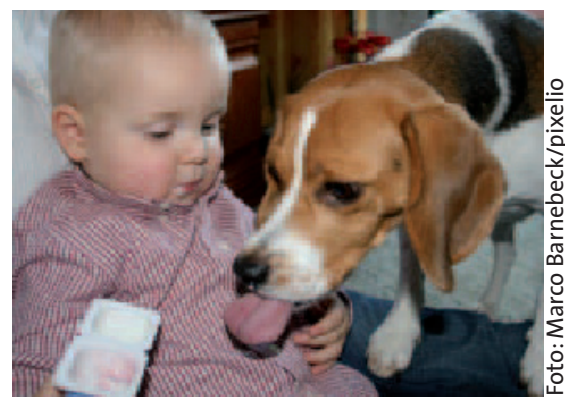

Hunde beschützen Kinder nicht nur vor bösen Menschen, sondern vielleicht auch vor Allergien.

\title{
Allergiegefahr durch Stents unklar
}

\section{Zur Behandlung von Gefäßverschlüssen bei koronarer Herzerkran- kung (KHK) werden oftmals Stents eingesetzt. Ein Problem ist die häufige Restenosierung, für die als Risikofaktor auch eine Allergie auf Stentmaterialien diskutiert wird.}

\footnotetext{
chwedische Forscher wollten einem Zusammenhang zwischen Stentimplantation und Allergieentwicklung auf die Spur kommen. Dazu unterzogen sie 484 Patienten, die einen koronaren Stent erhalten hatten, einem Patchtest und verglichen die Ergebnisse mit denen einer vergleichbaren entsprechenden Kontrollkohorte von 447 Dermatitispatienten. Die Stents enthielten Nickel oder waren goldbeschichtet.

Insgesamt 37\% der mit einem Goldstent versorgten Patienten wiesen eine Reaktion gegen Gold im Patchtest auf, in
}

der Kontrollkohorte waren es mit 19\% signifikant weniger $(p<0,001)$. Nickelallergien waren dagegen bei Patienten mit einem Nickelstent und den Kontrollen ähnlich häufig. Kontaktallergien auf Nickel und Gold waren unabhängig von der Art des verwendeten Stents bei den Herzpatienten gleich häufig. Bei den $\mathrm{Pa}-$ tienten mit Nickelallergie ergab sich eine klare Korrelation zwischen dem Vorhandensein von Zahngold und einer Goldallergie (Odds Ratio 3,2; p < 0,09). Nach Berücksichtigung der Präexposition mit Metallen wiesen denn auch die Pati- am deutlichsten, wenn der Hund bereits im ersten Lebensjahr Teil der Familie war. Allerdings fand sich im Alter von sechs Jahren kein Zusammenhang zwischen Hundehaltung und allergischen Symptomen sowie Erkrankungen. Der protektive Effekt hinsichtlich der Sensibilisierung gegen Aeroallergene wurde nur bei Hundehaltern ermittelt, nicht bei gelegentlichem Hundekontakt. Die Endotoxinbelastung im Alter von drei Monaten war im Gegensatz zur Hundeexposition nicht mit der Entwicklung von Sensibilisierungen assoziiert. Ebenso änderte die Berücksichtigung von Risikofaktoren wie Katzenhaltung oder Tabakrauch den Zusammenhang zwischen Hundehaltung und Sensibilisierungshäufigkeit nicht.

Fazit: Kinder, die aus Familien mit Hundehaltung kommen, weisen im Alter von sechs Jahren seltener Sensibilisierungen gegen Aeroallergene auf. Das hat allerdings keinen Effekt auf die Häufigkeit manifester allergischer Erkrankungen. $f k$

Chen CM et al. Dog ownership and contact during childhood and later allergy development. Eur Respir J 2008 Feb 6 [Epub ahead of print]

enten, die einen Goldstent erhalten hatten, nur noch einen Trend zu häufigeren Goldallergien auf, der Unterschied war nicht mehr statistisch signifikant. Angesichts dieses Ergebnisses warnen die Autoren davor, die häufigere Goldallergie bei Patienten mit Goldstents überzubewerten. Möglicherweise spielt die Alltagsexposition eine wichtigere Rolle bei der Entwicklung einer Goldallergie - lange vor der Stentimplantation.

Fazit: Zwar wurde bei Patienten, die einen Goldstent erhalten hatten, häufiger eine Kontaktallergie auf Gold gefunden. Es ist aber nicht auszuschießen, dass dies von einer früheren Exposition durch Zahngold oder Schmuck herrührt. $f k$

Ekqvist S et al. High frequency of contact allergy to gold in patients with endovascular coronary stents. Brit J Dermatol 2007; 157: 730-8 